\title{
Evaluation of the genotoxicity of zerumbone in cultured human peripheral blood lymphocytes.
}

\begin{abstract}
The chromosomal aberrations (CA) assay and micronucleus (MN) test were employed to investigate the effect in vitro of zerumbone (ZER) on human chromosomes. ZER is a sesquiterpene compound isolated from the rhizomes of wild ginger, Zingiber zerumbet Smith. The rhizomes of the plant are employed as a traditional medicine for some ailments and as condiments. ZER has been shown to have anti-cancer and apoptosis-inducing properties against various human tumour cells. It has also been shown to be active in vivo against a number of induced malignancies. Studies on ZER genotoxicity in cultured human peripheral blood lymphocytes (PBL) have not been reported so far. Therefore, the present study was undertaken to investigate the ability of ZER to induce chromosomal aberrations and micronuclei formation in human lymphocytes in vitro. Human blood samples were obtained from four healthy, non-smoking males aged 25-35 years. Cultures were exposed to the drug for $48 \mathrm{~h}$ at four final concentrations: 10, 20, 40 and $80 \mu \mathrm{M}$. Mitomycin C (MMC) was used as a positive control. The results of chromosomal aberrations assay showed that ZER was not clastogenic, when compared to untreated control, meanwhile MN test results showed a dosedependent increase in MN formation. The overall clastogenic effect of ZER on human PBL was statistically not significant. In conclusion, ZER is a cytotoxic but not a clastogenic substance in human PBL.
\end{abstract}

Keyword: Chromosome aberrations; Genotoxicity; Micronucleus; Zerumbone. 